and community health workers (without specific psychiatric training). In all categories, knowledge about mental health problems was regarded as poor. It is perhaps more surprising that similar results were obtained in a further survey of general practitioners (the vast majority of whom were seeing psychiatric patients).
Taken together, this set of papers emphasises the remarkable work being done by psychiatrists in some of the poorest countries in the world to provide better care for psychiatric patients, and stresses how important it is that they are supported in their endeavours by links with centres of excellence in the UK and elsewhere.

\title{
Management of mental health services in Malawi*
}

\section{Felix Kauye BSc(UK) MBBS(Malw) FCpsych(SA)}

Chief Government Psychiatrist, Ministry of Health, Zomba Mental Hospital, Malawi, email felixkauye@yahoo.com

$\mathrm{M}$ alawi is a country in sub-Saharan Africa bordering Mozambique, Tanzania and Zambia. It has an area of approximately $118000 \mathrm{~km}^{2}$ and is divided into northern, central and southern regions. It has an estimated population of 13 million, $47 \%$ of whom are under 15 years of age and just $5 \%$ over 60 years. Its economy is largely based on agriculture, with tobacco being the main export. The projected growth in gross domestic product (GDP) for 2007 was $8.8 \%$; GDP per capita was $\$ 284$ per annum.

The health system in Malawi is centrally controlled by the Ministry of Health. There are subsidiary zonal offices, which offer technical support to district health offices; the latter oversee the running of health services in all 28 districts. All but four of the 28 districts have their own district hospital. Within each district there are health centres, mainly manned by paramedics and nursing staff. These serve as referral centres for the health posts, which are manned by health surveillance assistants (HSAs). Most districts have at least one medical doctor, who usually performs administrative duties as a district health officer. There is a central hospital in each of the three regions; these act as tertiary units for the district hospitals in their regions. The directors of these central hospitals report directly to the Ministry of Health.

Mental health services in Malawi date from 1910. They were initially managed by the prison services, but in 1951 responsibility was transferred to the Ministry of Health. In 1953, a national psychiatric hospital was created in the southern region, in Zomba. It has about 333 beds and admits between 1500 and 2000 patients per year. Additional in-patient psychiatric facilities (run mainly by psychiatric nurses) are provided by the central hospital in the central region. In the northern region a missionary psychiatric unit is run by the Hospitaller Order of St John of God, which provides both community and in-patient services. (See Kauye \& Mafuta, 2006, for further details.)

Malawi, like most countries in this part of Africa, faces a number of problems in relation to the provision of mental

*This paper was presented to the African Division of the Royal College of Psychiatrists, June 2007. health services, including inadequate staffing levels, an overcentralisation of services and inconsistent drug supply. Over the years, it has looked at various ways of addressing these problems, which are reviewed here.

\section{The human resource problem}

Staffing problems have long affected all levels of service provision. At the Ministry of Health headquarters there is no national mental health coordinator to oversee activities; rather, Zomba Mental Hospital is currently coordinating mental health activities nationally. This has been successful to a certain extent, in that supervision of district mental health activities, revision of the Mental Health Act and preparation of an 'essential drug' policy for psychiatry have all been achieved. Nevertheless, a national coordinator at Ministry level is desirable.

Overall, there is a major shortage of clinicians with psychiatry as a specialty. District mental health services are mainly run by psychiatric nurses. Malawi has a general shortage of nursing staff, and although we have been training psychiatric nurses for a number of years, they end up doing general nursing duties and minimal mental healthcare. One way we are approaching this problem is by introducing a degree programme in mental health and clinical psychiatry for clinical officers with a diploma in clinical medicine. We hope clinical officers will be motivated to enrol as graduate students by the possibility of promotion on successful completion of the course. We are hoping that these graduates will mainly work in the districts and be at the forefront of promoting mental health, especially at community level.

The government of Malawi has been supplementing the salaries of health workers to a certain extent, in order to reduce their migration to developed countries. An improvement in the conditions of service of general health workers would influence mental health services both directly and indirectly. Having adequate numbers of nurses in the districts will mean that psychiatric nurses can be released to do more mental health activities. Sustaining the programme 
to supplement salaries in the longer term is something the government will have to consider.

\section{Centralisation of services}

Mental health services are very centralised and most districts treat few psychiatric patients. There is little community psychiatry done within districts. Mental health is not effectively integrated into primary healthcare, in contrast to our national mental health policy. Most primary healthcare workers do not feel competent and confident in dealing with psychiatric patients. Consequently, they refer nearly all psychiatric patients to the tertiary units, which are often far away. This makes it difficult for their relatives to visit them.

Plans have been made to train clinicians and nurses in all the districts of Malawi in the management of common mental health disorders, as effective integration of mental health services into primary care will mean that these disorders can be managed at district level by general medical staff. Two health workers from each district will undergo two weeks of intensive training using a special syllabus originally designed by the World Health Organization's collaborating centre at the Maudsley Hospital and Institute of Psychiatry; this syllabus has already been used for training equivalent workers in Kenya. The two health workers will then act as trainers for others in their respective districts, with support from the tertiary units. Once the training programme has been completed, we are hoping that the syllabus can be modified to be used for training of HSAs, so that they can include mental health as part of their activities in the community.

\section{Teaching of psychiatry}

Malawi has one medical school, with an intake of 60 students each year. It also has schools of health sciences, which train paramedics. The long-term sustainability of the decentralisation of mental health services will be achieved through good undergraduate teaching of psychiatry at both. The paramedics and general medical officers are the backbone of primary healthcare in Malawi.

The curriculum at the medical school has just been reviewed, and psychiatry teaching was increased from five to eight weeks. There are also plans to provide a standardised syllabus of psychiatry for all the schools of health sciences. Our teaching goals are being achieved through partnerships with institutions outside Malawi, including institutions in Scotland and Norway.

\section{Drug supply}

A shortage of drugs, including psychiatric medications, is one of the big challenges we face in Malawi. There are certain periods when most psychiatric medications are not available in the district and psychiatric hospitals. There are also problems with the procurement of drugs at national level. Central Medical Stores is the government department mandated with the procurement of drugs for government hospitals. This has faced a number of problems over the years and recently the government has engaged a consultancy firm from the USA to run the services in order to improve the situation.

We have incorporated psychiatric medications into the central procurement process. Estimates of drug requirements for all the district and tertiary mental health units have been submitted, based on figures from previous years. The plan is for Central Medical Stores to procure 6 months' supply in advance and to be given at least 2 months' notice when the drugs are about to run out. It is our hope that if this system is implemented, the supply of drugs will become more stable and patients will not have to go without them.

\section{Research}

Research on mental illness in Malawi is minimal. Most of it has been done through the work of the St John of God charitable service. Lack of funding opportunities and insufficient people with skills in research in mental health have contributed to this situation. Knowing that it can be a problem to get funding for big projects, Zomba Mental Hospital started an initiative whereby at least two small research projects are undertaken per year, using government funds. All cadres of staff, from junior nurses to psychiatrists, are encouraged to get involved. Small projects have been started in the past year, and we are also collaborating with overseas universities like Michigan State University in order to find funding for bigger research projects. We hope also to collaborate with universities in London and Manchester in this initiative of promoting mental health research in Malawi.

\section{Conclusion}

Staff shortages, over-centralisation of mental health services, inadequate drug supply and a lack of local data on mental health are some of the major challenges Malawi is trying to address in the context of its available resources. Decentralisation of services is closely linked to potential improvements in human resources at the district level. This need for decentralisation is being addressed in a number of ways, including the introduction of a degree programme for psychiatric clinical officers, strengthening of undergraduate teaching and training of primary healthcare workers in psychiatry using a special syllabus. Small-scale research projects using government funds are being done to fill some of the gaps in local data on mental illness, while bigger projects are being pursued in conjunction with universities outside Malawi. The government of Malawi has recently engaged a consultancy firm to address deficiencies in drug procurement and supply, which have been affecting all hospitals in Malawi, including psychiatric units, for a number of years. In the long term, it is important for countries like Malawi, with limited resources, to find sustainable solutions to run effective programmes for people with mental illness.

\section{Reference}

Kauye, F. \& Mafuta, C. (2006) Country profile: Malawi. International Psychiatry, 4, 9-11. 